\title{
Microscopic dynamics, chaos and transport in nonequilibrium processes
}

\author{
Santo Banerjee ${ }^{1,2, a}$ and Matteo Colangeli ${ }^{3}$ \\ ${ }^{1}$ Institute for Mathematical Research, Universiti Putra Malaysia, Selangor, Malaysia \\ ${ }^{2}$ Malaysia-Italy Centre of Excellence for Mathematical Science, Universiti Putra \\ Malaysia, Selangor, Malaysia \\ ${ }^{3}$ Dipartimento di Ingegneria e Scienze dell'Informazione e Matematica, Università di \\ L'Aquila, 67100 L' Aquila, Italy
}

Received 3 April 2019

Published online 30 May 2019

\begin{abstract}
This EPJ - Special Topics issue is a collection of contributions on theory and the recent developments in nonequilibrium statistical mechanics, fluctuations theory and dynamical systems. The various articles report results on the role of microscopic dynamics in giving rise to complex patterns observed at the macroscopic scale in a variety of physical phenomena.
\end{abstract}

Complexity, as per as information theory is concerned, may occur in various nonlinear real-world phenomena [1-3]. Like the human body, human heart is also a complex system, as it has emerging operations from the collective dynamics of millions of strongly interacting cells connected throughout the human body. A healthy heart is more complex than the same of a cardiac patient [3]. Another effective example is the communication networks (such as the Internet, which is a complex model), where complexity varies with different parameters, such as structure and evolution of the network, connection and node diversity, etc. As of late, it has been reported that, in the case of optical chaos communications (semiconductor lasers), complexity plays a crucial role in investigating the dynamics, instabilities, securities and output power, as it is strongly correlated with the output intensities. In particular, the study of microscopic dynamics through the prism of the analysis of complex phenomena has recently embraced a wide spectrum of applications, including kinetic and lattice gas modeling. A recent promising research line pointed toward the onset of "uphill currents" in particle and spin systems subject to a stochastic dynamics, in which much of the analytical effort has also been corroborated by Monte Carlo simulations, cf. e.g. [4-7]. The special issue on "Microscopic dynamics, chaos and transport in nonequilibrium processes" consists of 16 research articles. The articles can be grouped into six categories, namely,

- Fluid flow: heat and mass transfer

- Lattice models and stochastic dynamics

- Diffusion processes and phase transformations

- Microscopic chaos and chemical reactor

a e-mail: santoban@gmail.com 
- Fractals and applications

- Fractional model and associated dynamics.

In [8], the authors proposed a novel use of deterministic lateral displacement devices, where the separation of the particle suspension is performed under transient condition. Numerical results show that there exist device geometries and operating conditions where the dependence of the effective velocities can be exploited for performing a size-based separation of the dispersed suspension with a resolution that finds no counterpart in the corresponding time-continuous separation.

In [9], the authors proposed an analytical solution of a model describing the phase transition process in a metastable medium consists of the dimensionless heat/mass balance and kinetic equations. The analytical solution obtained shows that the transient phase transition process attains its steady-state solution at large times. The results can be used in different branches of theoretical and applied science like crystallization combined with polymerization, growth of protein and insulin aggregates, growth of particles in colloids and magnetic fluids, solidification with a mushy layer, dissolution of dispersed solids, evaporation of a polydisperse mist, combustion of a solid dispersed fuel, etc.

In [10], the authors presented numerical solutions for a coupled partial differential equations representing varying fluid properties on unsteady, free convective chemically reacting fluid flow on a moving vertical cone and flat plate. The influence of active parameters on transport properties of the fluid can be quantified using numerical results. It can be observed that the variable fluid properties have a significant influence on the flow, heat and mass transfer characteristics.

Section 2 is mainly on lattice model and stochastic dynamics, contains four articles [11-14]. In the analysis of lattice particle dynamics, the relevance of hyperbolic hydrodynamic modeling has been investigated. The morphology formation in a three state lattice system subjected to the evaporation of one component has also been observed.

In [15], the authors investigated analytically and numerically the effect of local thermodynamic equilibrium (LTE), for one-dimensional chains of $\mathrm{N}$ oscillators. It has been concluded that the standard laws and quantities of macroscopic systems are not appropriate to understand the behavior of such systems.

In [16], the authors investigate the effect of diffusion on varying the energy as a control parameter in a soft, periodic Lorenz gas. The different regimes have been investigated analytically in terms of simple random walk approximations.

In [17], the authors derived a new analytical solution of nonstationary integrodifferential coagulation equation, which describes the concluding stage of a phase transformation process in metastable liquids. The results can be applied to the growth of particulate aggregates in different physical systems.

Section 4 is about microscopic chaos and chemical reactors [18-20]. The authors proposed dynamical properties and complexity of chaotic chemical models with integer and fractional orders. In [19], the authors also investigated the chaotification technique that can be implemented on discrete population models.

Section 5 is on fractals and multifractals $[21,22]$. The authors have investigated several deterministic, random and stochastic fractals. The authors also investigated the morphology and fractals structures of polymer film by iterated function system (IFS) approach.

In [23], the authors presented the controllability results for Hilfer fractional stochastic system with multiple delays in control and Poisson jumps. The results have been verified with analytical and numerical investigations.

We would like to thank the authors for their contributions and the referees for their remarkable effort in reviewing the articles. We hope this issue will be helpful for the scientists and 
researchers working on the field of statistical mechanics and dynamical systems. Finally, we would like to thank all members of EPJ ST, for hosting this special issue, specially Dr. Caron, Sabine Lehr and Nicolas Puyaubreau for their kind support.

\section{References}

1. S. Mukherjee, S. Banerjee, L. Rondoni, Physica A 508, 131 (2018)

2. C.S. Poon, C.K. Merrill, Nature 389, 492 (1997)

3. M. Costa, A.L. Goldberger, C.-K. Peng, Phys. Rev. Lett. 71, 021906 (2005)

4. M. Colangeli, A. De Masi, E. Presutti, Phys. Lett. A 380, 1710 (2016)

5. M. Colangeli, A. De Masi, E. Presutti, J. Stat. Phys. 167, 1081 (2017)

6. A. De Masi, E. Presutti, D. Tsagkarogiannis, Arch. Ration. Mech. Anal. 201, 681 (2011)

7. E. Presutti, Scaling Limits in Statistical Mechanics and Microstructures in Continuum Mechanics, Theoretical and Mathematical Physics (Springer, Berlin, 2009)

8. M.A. Murmura, A. Adrover, S. Cerbelli, Eur. Phys. J. Special Topics 228, 5 (2019)

9. E. Makoveeva, D. Alexandrov, Eur. Phys. J. Special Topics 228, 25 (2019)

10. R. Sivaraj, A. Jasmine Benazir, S. Srinivas, A.J. Chamkha, Eur. Phys. J. Special Topics 228, 35 (2019)

11. E.N.M. Cirillo, M. Colangeli, E. Moons, A. Muntean, S.-A. Muntean, J. van Stam, Eur. Phys. J. Special Topics 228, 55 (2019)

12. M. Colangeli, C. Giberti, C. Vernia, M. Kröger, Eur. Phys. J. Special Topics 228, 69 (2019)

13. A. Brasiello, D. Cocco, F. Garofalo, M. Giona, Eur. Phys. J. Special Topics 228, 93 (2019)

14. A. Ciallella, E.N.M. Cirillo, Eur. Phys. J. Special Topics 228, 111 (2019)

15. C. Giberti, L. Rondoni, C. Vernia, Eur. Phys. J. Special Topics 228, 129 (2019)

16. S.S. Gil Gallegos, R. Klages, J. Solanpää, E. Räsänen, Eur. Phys. J. Special Topics 228, $143(2019)$

17. D.V. Alexandrov, A.A. Ivanov, I.V. Alexandrova, Eur. Phys. J. Special Topics 228, 161 (2019)

18. P.P. Singh, B. Krishna Roy, Eur. Phys. J. Special Topics 228, 169 (2019)

19. H. Natiq, S. Banerjee, M.R.M. Said, Eur. Phys. J. Special Topics 228, 185 (2019)

20. S. He, S. Banerjee, K. Sun, Eur. Phys. J. Special Topics 228, 195 (2019)

21. M.D. Hassan, Eur. Phys. J. Special Topics 228, 209 (2019)

22. C. Raja Mohan, A. Gowrisankar, R. Uthayakumar, K. Jayakumar, Eur. Phys. J. Special Topics 228, 233 (2019)

23. T. Sathiyaraj, P. Balasubramaniam, Eur. Phys. J. Special Topics 228, 245 (2019) 